\title{
Cross-Cross Resonance Gate
}

\author{
Kentaro Heya ${ }^{1,2,{ }^{*}}$ and Naoki Kanazawa ${ }^{1, \dagger}$ \\ ${ }^{1}$ IBM Quantum, IBM Research Tokyo, 19-21 Nihonbashi Hakozaki-cho, Chuo-ku, Tokyo 103-8510, Japan \\ ${ }^{2}$ Research Center for Advanced Science and Technology (RCAST), The University of Tokyo, Meguro-ku, Tokyo \\ 153-8904, Japan
}

(Received 9 June 2021; accepted 19 October 2021; published 16 November 2021)

\begin{abstract}
The implementation of high-fidelity swapping operations is of vital importance for executing quantum algorithms on a quantum processor with limited connectivity. We present an efficient pulse-control technique, the cross-cross resonance (CCR) gate, to implement isWAP and SWAP gates with dispersively coupled fixed-frequency transmon qubits. The key to the operation of the CCR gate is the simultaneous driving of both of the coupled qubits at the frequency of another qubit, whereby a fast two-qubit interaction roughly equivalent to $Z X+X Z$ entangling gates is realized without strongly driving the qubits. We develop a calibration technique for the CCR gate and evaluate the performance of the isWAP and SWAP gates. The CCR gate shows remarkable improvements in the average gate error from the conventional decomposition based on the cross-resonance gate.
\end{abstract}

DOI: 10.1103/PRXQuantum.2.040336

\section{INTRODUCTION}

The number of qubits available in a superconducting quantum processor has been continuously growing despite the restricted connectivity exhibited by square and heavyhexagon lattice structures [1]. The connections in these structures are carefully designed to avoid overlapping transition frequencies with neighboring qubits while enabling the implementation of an error-correction code. This is the key ingredient of fault-tolerant quantum processors [2-7]. Although such processors can implement state-of-the-art two-qubit entangling gates with an average gate error of less than $10^{-2}$ [8], the insertion of multiple swapping operations to entangle nonconnected qubit pairs becomes a visible obstacle to building large-scale quantum processors [9]. Therefore, further improvement of entangling gates is indispensable.

With superconducting qubits, entangling gates are generally implemented using electric dipole interactions between qubits, and can be roughly divided into two subclasses. One class of implementations is realized by dynamically modulating the device parameters to switch the coupling with the aid of tunable elements [10-13].

\footnotetext{
*kheya@qc.rcast.u-tokyo.ac.jp

†knzwnao@jp.ibm.com

Published by the American Physical Society under the terms of the Creative Commons Attribution 4.0 International license. Further distribution of this work must maintain attribution to the author(s) and the published article's title, journal citation, and DOI.
}

The additional control lines for the tunable elements may induce an extra degree of interaction with a noisy environment, and such an architecture tends to show shorter coherence times. Another class of implementations uses the manipulation of Hamiltonian terms by irradiating a pair of dispersively coupled qubits with microwave pulses [14-19]. Such architectures can be realized without tunable elements. Thus, qubits can be well protected from the source of decoherence at the cost of a longer gate time [20], impeded by a crosstalk error due to the static $Z Z$ interaction [21,22].

The cross-resonance (CR) gate is a typical implementation of the latter subclass, without any tunable elements $[14,20,23]$. In the CR gate, a $Z X$ interaction is induced by irradiating one of the two coupled qubits with microwave radiation that resonates with the other qubit. This simple architecture offers benefits for the scaling up of devices $[14,20,23]$. However, in the CR gate, there is a trade-off between fast gate execution and adherence to adiabatic conditions. For fast gate execution, we need to increase the microwave pulse intensities, which makes the adiabatic condition break, resulting in adverse effects such as driveinduced saturation and population leakage to higher-order levels [24,25].

In this paper, we propose a pulse sequence, called the cross-cross resonance (CCR) gate, which relaxes the above trade-off. The CCR gate is a natural extension of the $\mathrm{CR}$ gate, where a $Z X+X Z$ interaction is induced by simultaneously irradiating both of the two coupled qubits with microwaves that resonate with the other qubit. Although both qubits are driven simultaneously, the 
required microwave intensities in the CCR gate are weaker than those in CR gates. Thus, the CCR gate avoids the adverse effects derived from strong drives while preserving the gate execution time.

This paper is organized as follows. In Sec. II, we first show the advantage of CCR gates over CR gates by theoretical calculations. In Sec. III, we next demonstrate a CCR gate on the IBM Quantum cloud provider using Qiskit Pulse [26,27]. The calibration of the CCR gate is scrutinized, and we introduce a channel purification technique that enables the investigation of an approximate unitary representation of experimental gates. The calibrated CCR gate demonstrates remarkable improvements in the average gate fidelity, which is confirmed by interleaved randomized benchmarking [28-30].

\section{PRINCIPLE}

We describe a model of the CCR gate using the Hamiltonian of the standard circuit-quantum-electrodynamics setup. A system consisting of two qubits dispersively coupled to a bus resonator can be modeled using Duffing oscillators:

$$
\begin{aligned}
\mathcal{H}= & \sum_{i \in[0,1]}\left\{\tilde{\omega}_{i} b_{i}^{\dagger} b_{i}+\frac{\alpha_{i}}{2} b_{i}^{\dagger} b_{i}^{\dagger} b_{i} b_{i}\right\} \\
& +g\left(b_{0}^{\dagger}+b_{0}\right)\left(b_{1}^{\dagger}+b_{1}\right) \\
& +\sum_{i \in[0,1]} \Omega_{i} \cos \left(\omega_{d i} t\right)\left(b_{i}^{\dagger}+b_{i}\right),
\end{aligned}
$$

where $b_{i}\left(b_{i}^{\dagger}\right)$ is the annihilation (creation) operator, and $\tilde{\omega}_{i}, \alpha_{i}$, and $g$ are the dressed eigenfrequency, the anharmonicity of the $i$ th qubit, and the coupling between the two qubits, respectively. The last term describes the general drive Hamiltonian for each qubit, with a time-invariant drive $\Omega_{i}$ and drive frequency $\omega_{d i}$. Let $i \in[0,1]$ denote the labels for the control and target qubits, respectively.

\section{A. Cross-resonance gate}

We start with the Hamiltonian of the CR gate, which is the predecessor of our proposal. The $\mathrm{CR}$ gate is a microwave-only two-qubit entangling gate that is commonly used for fixed-frequency dispersively coupled qubits [23]. In the CR gate, the control qubit is irradiated with a microwave tone at the frequency of the target qubit $\omega_{d 0}=\tilde{\omega}_{1} \sim \omega_{1}-g^{2} / \Delta$, where $\Delta=\tilde{\omega}_{0}-\tilde{\omega}_{1}$ is the detuning of the two qubits. This stimulus induces a controlled rotation of the target qubit, whose direction depends on the state of the control qubit.

For simplicity, we model transmons as ideal qubits, ignoring classical crosstalk [20]. The effective Hamiltonian of the CR gate for a qubit model under the rotating-wave approximation $\Omega_{0} /\left(\tilde{\omega}_{0}+\tilde{\omega}_{1}\right) \ll 1$, the strong dispersive condition $g / \Delta \ll 1$, and the weak-drive condition $\Omega / \Delta \ll$ 1 is written as follows [31]:

$$
\mathcal{H}_{\mathrm{eff}}^{\mathrm{CR}} \sim \frac{\operatorname{sgn}(\Delta) g \Omega_{0}}{\sqrt{\Delta^{2}+\Omega_{0}^{2}}} \frac{Z X}{2},
$$

where $Z X$ denotes the tensor product of the standard single-qubit Pauli operators $Z$ and $X$. Note that the above notation for the effective Hamiltonian $\mathcal{H}_{\text {eff }}$ is a representation in a rotating frame moved from the laboratory frame using the unitary operator

$$
R=e^{-i\left(\Omega_{0}^{2} / 2 \Delta\right) t(Z I / 2)} .
$$

To clarify the difference between CR and CCR gates, we characterize the two-qubit gate implemented by each pulse sequence using the KAK decomposition [32]. Any twoqubit gate can be described as a $4 \times 4$ matrix in SU(4), which can be decomposed as follows:

$$
U=k_{1} A k_{2},
$$

where $k_{1}$ and $k_{2}$ are $4 \times 4$ matrices in $\mathrm{SU}(2) \otimes \mathrm{SU}(2)$, and $A$ is a $4 \times 4$ matrix in the maximal Abelian subgroup $\mathcal{A}$ of $\mathrm{SU}(4)$. The matrix $A$ is parameterized as follows:

$$
A\left(c_{1}, c_{2}, c_{3}\right)=\exp \left(-i \sum_{i=1}^{3} c_{i} \frac{A_{i}}{2}\right),
$$

where $c_{1,2,3} \in[0, \pi / 2]$ are the Cartan coefficients, and $A_{1,2,3}$ is any combination of mutually commutative two-qubit Pauli operators, such as $\{X X, Y Y, Z Z\}$ and $\{Z X, X Z, Y Y\}$. The controlled-NOT (CNOT), isWAP, and SWAP gates, which are typical two-qubit gates, have Cartan coefficients $(\pi / 2,0,0),(\pi / 2, \pi / 2,0)$, and $(\pi / 2, \pi / 2, \pi / 2)$, respectively. These coefficients illustrate the overhead of three CNOT gates required for the decomposition of the SWAP gate. The CR gate has Cartan coefficients as follows:

$$
\boldsymbol{c}(t)=\left(\frac{\operatorname{sgn}(\Delta) g \Omega_{0}}{\sqrt{\Delta^{2}+\Omega_{0}^{2}}} t, 0,0\right),
$$

which is locally equivalent to a controlled-rotation gate, namely the fundamentals of the CNOT gate. Note that without the qubit approximation, terms derived from higherorder levels are added to the effective Hamiltonian [31].

\section{B. Cross-cross resonance gate}

The CCR gate is a natural extension of the CR gate, where the control and target qubits are simultaneously irradiated with microwave tones at the frequency of the other qubit, and $\omega_{d 0}=\tilde{\omega}_{1}-\Omega_{0}^{2} / 2 \Delta$ and $\omega_{d 1}=\tilde{\omega}_{0}+\Omega_{1}^{2} / 2 \Delta$. In 
contrast to the CR gate, the drive frequencies of the CCR gate are a function of the drive amplitudes $\Omega_{0}$ and $\Omega_{1}$ owing to the drive-induced Stark shift $[33,34]$. We again use the qubit model for simplicity, and find that the effective Hamiltonian of the CCR gate can be described as follows:

$\mathcal{H}_{\mathrm{eff}}^{\mathrm{CCR}} \sim \frac{\operatorname{sgn}(\Delta) g \Omega_{0}}{\sqrt{\Delta^{2}+\Omega_{0}^{2}+\frac{3}{2} \Omega_{1}^{2}}} \frac{Z X}{2}-\frac{\operatorname{sgn}(\Delta) g \Omega_{1}}{\sqrt{\Delta^{2}+\frac{3}{2} \Omega_{0}^{2}+\Omega_{1}^{2}}} \frac{X Z}{2}$.

Given $\Omega_{1}=0$, the effective Hamiltonian of the CCR gate matches that of the CR gate shown in Eq. (2). Details are shown in Appendix A. The CCR gate has the following Cartan coefficients:

$$
c(t)=\left(\frac{+\operatorname{sgn}(\Delta) g \Omega_{0}}{\sqrt{\Delta^{2}+\Omega_{0}^{2}+\frac{3}{2} \Omega_{1}^{2}}} t, \frac{-\operatorname{sgn}(\Delta) g \Omega_{1}}{\sqrt{\Delta^{2}+\frac{3}{2} \Omega_{0}^{2}+\Omega_{1}^{2}}} t, 0\right) .
$$

\section{Effective Hamiltonian of CR and CCR gates with higher-order excited levels}

Next, we discuss effects derived from higher-order excited levels. For simplicity, we deal only with the leading-order terms up to first order in $g / \Delta$ and $\Omega / \Delta$. The effective Hamiltonian of the CR gate in the qutrit system [31] is known to be as follows:

$$
\mathcal{H}_{\mathrm{eff}}^{\mathrm{CR}}=\frac{g \Omega_{0}}{\Delta}\left(\frac{\alpha_{0}}{\alpha_{0}+\Delta}\right) \frac{Z X}{2}+\frac{g \Omega_{0}}{\Delta}\left(\frac{\Delta}{\alpha_{0}+\Delta}\right) \frac{I X}{2},
$$

where an $I X$ term, called the quantum crosstalk, appears and can be canceled by applying a cancellation drive to the target qubit [20]. On the other hand, the effective Hamiltonian of a CCR gate with cancellation drives can be calculated as follows:

$$
\mathcal{H}_{\mathrm{eff}}^{\mathrm{CCR}}=\frac{g \Omega_{0}}{\Delta}\left(\frac{\alpha_{0}}{\alpha_{0}+\Delta}\right) \frac{Z X}{2}-\frac{g \Omega_{1}}{\Delta}\left(\frac{\alpha_{1}}{\alpha_{1}-\Delta}\right) \frac{X Z}{2},
$$

which is a simple sum of the effective Hamiltonians of bidirectional $\mathrm{CR}$ gates with cancellation drives. In the case of the qutrit model, the anharmonicity of each qutrit appears in the coefficients of the $Z X$ and $X Z$ terms, indicating that we should irradiate the two qubits with different drive amplitudes.

\section{Advantages of CCR gates over CR gates}

In what follows, we compare CCR and $\mathrm{CR}$ gates in terms of drive-induced saturation and leakage from the computational basis.

\section{Tolerance to drive-induced saturation}

As one can see from Eqs. (2) and (7), the strength of the entanglement-generation term is a linear function of the drive strength under the weak-drive condition, but it is known to be saturated by third-order terms when the drive amplitude becomes comparable to the detuning between the qubits. This phenomenon is one of the adverse effects originating from the violation of the adiabatic condition, and we call it drive-induced saturation. In CCR gates, the total power of the microwave drive is split between the qubits, and they are driven far below the saturation point. In other words, we can further reduce the gate time of CCR gates within a limited power budget. The gate times required to compose the SWAP operation are estimated as

$$
t_{\mathrm{SWAP}}^{\mathrm{CR}}=\frac{3 \pi \sqrt{\Delta^{2}+\Omega^{2}}}{2 g \Omega}, t_{\mathrm{SWAP}}^{\mathrm{CCR}}=\frac{3 \pi \sqrt{\Delta^{2}+\frac{5}{8} \Omega^{2}}}{2 g \Omega},
$$

where we assume a CCR drive amplitude $\Omega_{0}=\Omega_{1}=$ $\Omega / 2$, which is equivalent to a CR drive amplitude $\Omega$, for the sake of a fair comparison between the two entangling gates. Because $\Delta^{2}, \Omega^{2}>0$, we find that $t_{\mathrm{SWAP}}^{\mathrm{CR}}>$ $t_{\mathrm{SWAP}}^{\mathrm{CCR}}$; hence, the CCR gate can always implement faster swapping operations at the same drive strength.

\section{Tolerance to leakage}

Leakage from the computational basis is also one of the typical error sources in CR gates. To execute a fast $\mathrm{CR}$ gate, we need to increase the CR drive amplitude, which often excites the control qubit to higher-order levels. Therefore, leakage is a factor that often limits the execution speed of a CR gate. Similarly to drive-induced saturation, leakage from the computational basis is also a phenomenon originating from higher-order terms concerning drive amplitudes, and it is easy to expect that CCR gates will have a higher tolerance to this leakage than CR gates. We calculate the optimal CCR drive amplitude ratio that maximizes the gate execution speed of isWAP and SWAP gates while suppressing the leakage as follows:

$$
\frac{\Omega_{1}}{\Omega_{0}}=\frac{\alpha_{1}\left(\alpha_{1}-\Delta\right)}{\alpha_{0}\left(\alpha_{0}+\Delta\right)} .
$$

Based on this condition, we compare the maximum amount of leakage in CCR and CR gates in Appendix B. Provided that the anharmonicities of the two qubits are comparable and the gates are calibrated at the same execution time, we find that CCR gates induce leakage less than that in CR gates by a factor of 2 .

\section{EXPERIMENT}

\section{A. System}

The experiments presented in this paper are executed on qubits $Q 3$ and $Q 4$ of the five-qubit system ibmq_bogota 
at IBM Quantum. This processor consists of dispersively coupled fixed-frequency transmon qubits, and the control electronics are capable of generating arbitrary waveforms with a cycle time $d t=0.222 \mathrm{~ns}$, afforded by a fast digitalto-analog converter. The device parameters at the time of the experiment are shown in Table I.

The coupling strength between the two qubits is found to be $g / 2 \pi=1.40 \mathrm{MHz}$. Because the CCR gate drives the cross-resonance from both directions, the drive Hamiltonian consists of multiple frequency components. To realize a high-fidelity CCR gate, all transition frequencies, including higher energy levels, must be well isolated [1]. The selected qubit pair satisfies this requirement. Thus, it is considered to be a suitable experimental platform for our demonstration.

First, we independently calibrate a CR pulse with $Q 3$ as the control and $Q 4$ as the target qubit, and vice versa. The CR pulse envelopes with which $Q 3$ and $Q 4$ are irradiated correspond to $R_{Z X}(\pi / 4)=e^{-i(\pi / 8) Z X}$ and $R_{X Z}(\pi / 4)=e^{-i(\pi / 8) X Z}$ gates, respectively. Both CR pulse envelopes are "Gaussian square" pulses, that is, square pulses with Gaussian-shaped rising and falling edges. In addition, both pulses have a total duration of $t_{\mathrm{CR}}=$ $848 d t=188.4 \mathrm{~ns}$. The square portion of each of the pulses has a duration of $592 \mathrm{dt}$, and the Gaussian rising and falling edges last $128 d t$ and have a standard deviation of $64 d t$. In ibmq_bogota, a standard CR gate is calibrated with a rotary echo, which eliminates almost all unwanted dynamics by driving the target qubit with an intense microwave tone at the resonance frequency. However, this technique cannot be applied to the CCR gate, because the induced local Hamiltonian terms $I X+X I$ may suppress the generator terms $X Z+Z X$ of the gate. For this reason, we calibrate the $\mathrm{CR}$ gates with active cancellation, wherein a target qubit is driven by a weak resonance tone in the opposite phase to the unwanted local Hamiltonian dynamics represented by $I X+I Y$ [20]. The CR gates are carefully tuned using the selective Pauli erroramplifying technique that we recently developed [35]; this is akin to the technique of Hamiltonian error-amplifying tomography (HEAT) [36]. We should keep in mind that unwanted dynamics represented by a nonlocal $Z Z$ interaction may still be alive and contribute slightly to the dynamics of CCR gates. See Appendix E for a detailed analysis.

TABLE I. Parameters of qubits.

\begin{tabular}{lcc}
\hline \hline & $Q 3$ & $Q 4$ \\
\hline$\omega / 2 \pi$ & $4.858 \mathrm{GHz}$ & $4.978 \mathrm{GHz}$ \\
$\alpha / 2 \pi$ & $-324 \mathrm{MHz}$ & $-338 \mathrm{MHz}$ \\
$T_{1}$ & $112.4 \mu \mathrm{s}$ & $115.5 \mu \mathrm{s}$ \\
$T_{2}$ & $191.7 \mu \mathrm{s}$ & $167.6 \mu \mathrm{s}$ \\
\hline \hline
\end{tabular}

\section{B. Calibration of drive frequencies}

Next, we calibrate the detuning of each microwave-tone frequency to realize the $\mathrm{CCR}$ gate by fixing the tone amplitude at the optimal point of the underlying CR gates. As mentioned in Sec. B, the existence of higher-order levels changes the frequency shift owing to the off-resonance microwave drives. Therefore, we need to simultaneously calibrate two drive frequencies, $\omega_{d 0}$ and $\omega_{d 1}$. In the following, we change the notation of the qubit from $i=0$ (1) to 3 (4) to clarify the correspondence with physical qubits. Note that we apply an active cancellation tone for another $\mathrm{CR}$ drive along with the $\mathrm{CR}$ tone for the qubit, which usually differs by a frequency of approximately $\Delta /(2 \pi)$.

Figure 1 shows the experimental [Fig. 1(a)] and numerical [Fig. 1(b)] excited-state populations of the qubits after the simultaneous excitation while the drive frequencies are swept, where the excited-state population refers to the probability of existence in states other than the ground state. In our numerical simulation, we treat a transmon as a three-level system and apply the parameters shown in Table I, which are obtained in preliminary experiments. The experimental result is in qualitatively good agreement with the simulation result. The optimal detuning frequencies are highlighted by a star symbol where the two CR transitions cross over. This point is also experimentally confirmed to be at $\left(\omega_{d 4}-\tilde{\omega}_{3}\right) / 2 \pi=-18.0 \mathrm{MHz}$, $\left(\omega_{d 3}-\tilde{\omega}_{4}\right) / 2 \pi=8.9 \mathrm{MHz}$.

In addition to the $\mathrm{CR}$ dynamics, several unwanted transitions are also confirmed experimentally. The $g-f$ transition of $Q 4$ indicates the frequency of resonance with the nonlinear two-photon transition process $|g g\rangle \rightarrow|g f\rangle$ occurring at $\tilde{\omega}_{4}+\alpha_{4} / 2$, which is approximately $-50 \mathrm{MHz}$ from $\tilde{\omega}_{3}$. The $g$-e transition $|g g\rangle \rightarrow|g e\rangle$ of this qubit is found at $\tilde{\omega}_{4} \sim \tilde{\omega}_{3}+\Delta$. The $g-e$ transition $|g g\rangle \rightarrow|e g\rangle$ of $Q 3$ is observed at $\tilde{\omega}_{3} \sim \tilde{\omega}_{4}-\Delta$. We also find a two-photon blue-sideband transition in which $|g g\rangle \rightarrow|e e\rangle$ is driven at $\tilde{\omega}_{3}+\tilde{\omega}_{4} / 2$, and this appears as yellow trajectories [37]. The optimal driving point is sufficiently isolated from these transitions, which indicates that we can generate a stable CCR gate with $Q 3$ and $Q 4$. See Appendix C for details.

\section{Calibration of gate time}

As previously discussed, it is not efficient to control the time evolution of the CCR gate with the pulse amplitude because the calibrated frequency offset, which is induced by the ac Stark effect, is a function of the drive amplitude. Thus, we calibrate the target gate by scanning over the gate time to find the optimal value, at which we realize an entangling gate almost locally equivalent to a $\sqrt{\text { iSWAP }}$ gate, which is a perfect entangler [32]. Owing to the nonzero $Z Z$ interaction, a single CCR gate does not generate an entangling gate that is locally equivalent an iswAP-type gate. 
(a)

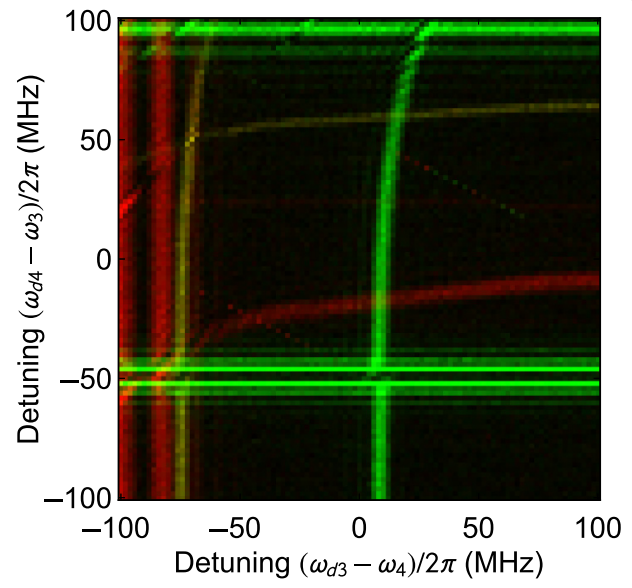

(b)

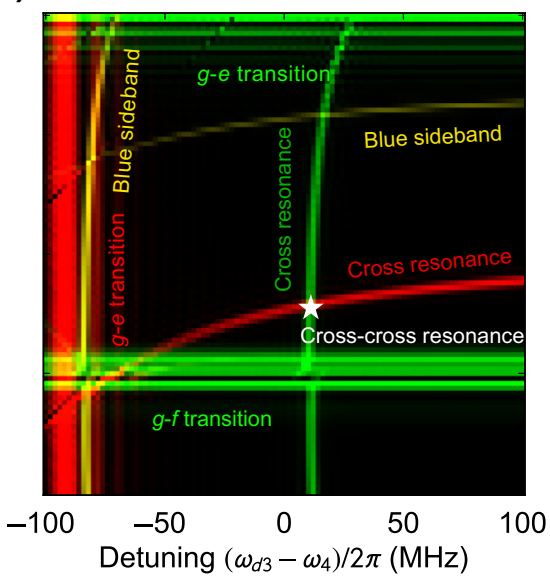

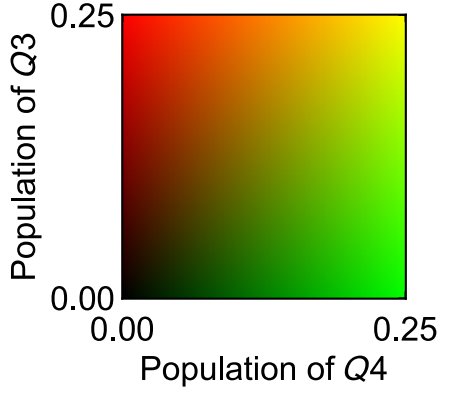

Population of $Q 4$

FIG. 1. Heatmaps of qubit excited-state population measured after CCR gates with various frequency detunings from the predetermined drive frequencies based on the individual CR gate calibration. (a) Experimental results and (b) simulation results. The measured populations of $Q 3$ and $Q 4$ are overlaid in the same plot to highlight an optimal driving point. The excited-state populations of $Q 3$ and $Q 4$ correspond to red and green, respectively. The yellow region indicates frequencies at which both $Q 3$ and $Q 4$ are simultaneously excited. See the discussion in the main text. In each panel, the vertical (horizontal) axis represents the detuning from the predetermined CR frequency of $Q 4(Q 3)$, corresponding to the frequency of the target qubit $Q 3(Q 4)$. The labels in (b) indicate the origin of each transition.

However, as is described later in Sec. III D, this interaction can be nullified with multiple CCR gates composed of iSWAP or SWAP gates. See Appendix E for details.

The target CCR gate is found when the following conditions for the Cartan coefficients are satisfied:

$$
\begin{aligned}
c_{1}(t)+c_{2}(t) & =\frac{\pi}{2}, \\
c_{3}(t) & =0 .
\end{aligned}
$$

To this end, we need to measure the Cartan coefficients while sweeping the CCR gate time. The evolution of a quantum system can be investigated using quantum process tomography (QPT) with measurement-error mitigation [38-41], which extracts a superoperator representation of a quantum process by tomographic reconstruction [42]. Because the Cartan coefficients are defined only on the unitary-matrix representation, we extract a unitary matrix from a superoperator estimated from a QPT experiment with the aid of a channel purification protocol. This technique is detailed in Appendix D. Figure 2(b) shows the approximate Cartan coefficients of the entangling gates while the total duration of the CCR drive is swept from $256 d t$ to $1600 d t$ with fixed rising and falling edges. It is found that two Cartan coefficients increase at the same rate and one remains at zero, as predicted by our effectiveHamiltonian model shown in Eq. (11). This indicates that the CCR drive generates entangling gates in the $X Y$ interaction family, in which the isWAP gate belongs [43]. From Fig. 2(b), we find the optimal total duration of the CCR drive to be $976 d t=216.9 \mathrm{~ns}$. (a)

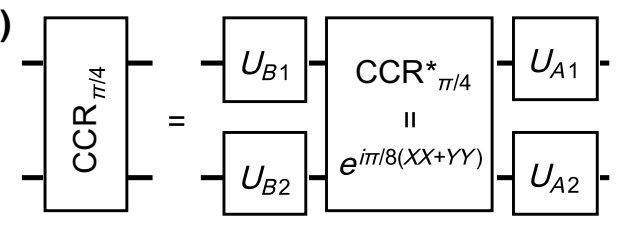

(b)

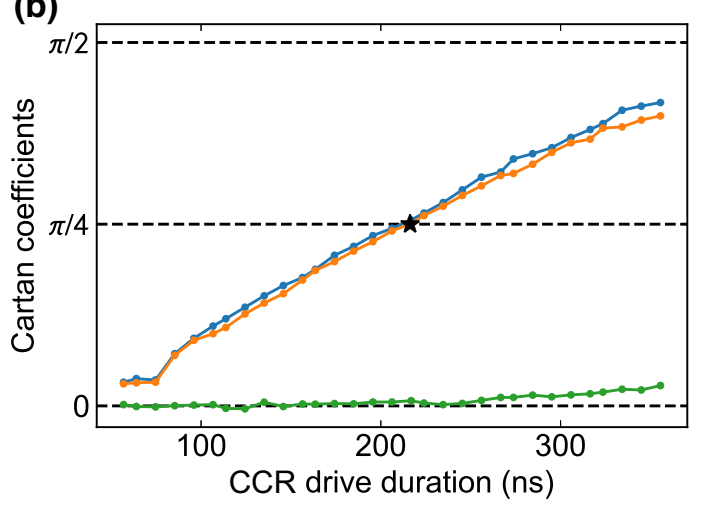

FIG. 2. (a) Desired condition for an entangling gate generated by CCR drive. (b) Approximate Cartan coefficients of entangling gates while the CCR drive duration is swept.

As shown in Fig. 2(a), the unitary matrix corresponding to the CCR drive with the optimal time can be decomposed as follows:

$$
R_{\mathrm{CCR}_{\pi / 4}}=\left(U_{A 1} \otimes U_{A 2}\right) R_{\mathrm{CCR}_{\pi / 4}^{*}}\left(U_{B 1} \otimes U_{B 2}\right),
$$


where $U_{A 1, A 2, B 1, B 2}$ represents a $2 \times 2$ matrix, and $\mathrm{CCR}_{\pi / 4}^{*}$ represents a $4 \times 4$ matrix in the maximum Abelian subgroup of $\mathrm{SU}(4)$.

\section{Two-qubit randomized benchmarking with CCR gates}

Finally, we implement iSWAP and SWAP gates consisting of multiple CCR gates and compare the performance of these gates with the standard gate decomposition based on CR gates. This is supported by the IBM Quantum cloud provider. We have already obtained an expression for the local rotations $U_{A 1, A 2, B 1, B 2}$ by the KAK decomposition of $\mathrm{CCR}_{\pi / 4}$. Therefore, we can generate $\mathrm{CCR}_{\pi / 4}^{*}$ gates as follows:

$$
R_{\mathrm{CCR}_{\pi / 4}^{*}}=\left(U_{A 1}^{\dagger} \otimes U_{A 2}^{\dagger}\right) R_{\mathrm{CCR}_{\pi / 4}^{*}}\left(U_{B 1}^{\dagger} \otimes U_{B 2}^{\dagger}\right)
$$

As shown in Figs. 3(a) and 3(b), we can implement the isWAP and SWAP gates with echo sequences as follows:

$$
\begin{aligned}
& U_{\mathrm{iSWAP}}=\left(R_{\mathrm{CCR}_{\pi / 4}^{*}} R_{\mathrm{XY}} \otimes R_{\mathrm{XY}}\right)^{2}, \\
& U_{\mathrm{SWAP}}=\left(R_{\mathrm{CCR}_{\pi / 4}^{*}} R_{\mathrm{XYZ}} \otimes R_{\mathrm{XYZ}}\right)^{3},
\end{aligned}
$$

where $R_{\mathrm{XY}}=e^{i(\pi / 2)(X+Y / \sqrt{2})}$ and $R_{\mathrm{XYZ}}=e^{i(\pi / 3)(X+Y+Z / \sqrt{3})}$ represent single-qubit gates. The echoes are designed to circumvent the gate errors caused by the $Z Z$ interaction derived from higher-order levels. Details are shown in Appendix E. On the other hand, the isWAP and SWAP gates are also decomposed into two and three two-pulseechoed Controlled-X (TPCX) gates, respectively, which are echoed versions of the CR gate [2]. In our setup, the TPCX gate is calibrated at a gate time of $334.2 \mathrm{~ns}$. On the other hand, the CCR gate is calibrated at $216.9 \mathrm{~ns}$, which is restricted by the slow speed of the interaction from $Q 3$ to $Q 4$.

Because the iSWAP and SWAP gates belong to the Clifford group, the performance of the gates can be precisely measured with a two-qubit interleaved randomized benchmarking (IRB) experiment. Figures 3(c) and 3(d) show the experimental results from an IRB experiment. We create ten random circuits for each Clifford-sequence length and take 1024 samplings of measurements for each random circuit to obtain a data point. The curves of the population other than that in the ground state are fitted by an exponential decay with the same scale and offset parameters in each figure. The performance of these gates and the coherence limits calculated from the calibrated gate time and from coherence times measured for idling qubits are summarized in Table II. From the table, it is found that the average gate infidelities obtained from IRB do not reach the coherence limits. However, it should be noted that coherence times during gate execution are generally (a)
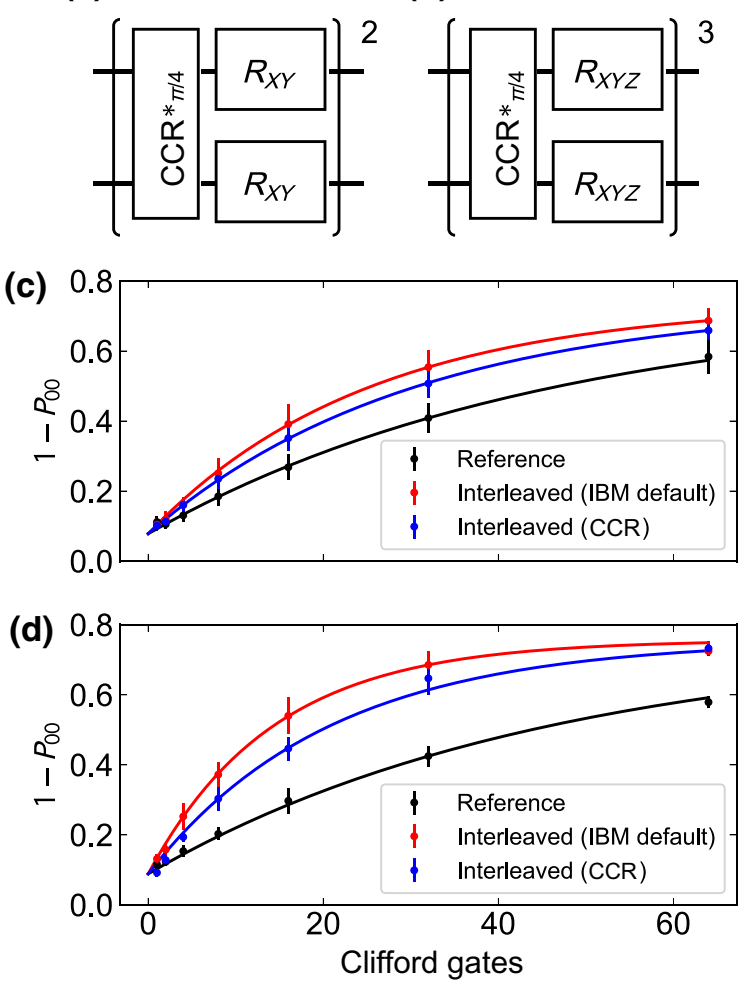

FIG. 3. (a) Gate sequence to generate isWAP gate with two CCR gates. (b) Gate sequence to generate SWAP gate with three CCR gates. Experimental results from two-qubit interleaved randomized benchmarking for (c) isWAP and (d) SWAP gates with the gate decomposition based on CR gates, supported by the IBM Quantum cloud provider, and with the gate decomposition based on CCR gates.

different from premeasured values due to the microwave irradiation [44-46].

To extract the contribution of the unitary error from the total error of the iSWAP and SWAP gates generated with CCR gates, we carefully analyze the Pauli transfer matrices previously measured by QPT experiments, as shown in Fig. 4(a). From the product of the inverse Pauli transfer matrices of the ideal gate and those obtained from the QPT experiments, we obtain the Pauli transfer matrices of the gate error. By applying dynamical generator analysis [47] to them, the unitary-matrix representations of the gate

TABLE II. Comparison of performance of isWAP and SWAP gates implemented with CR and CCR gates.

\begin{tabular}{lccccc}
\hline \hline & \multicolumn{2}{c}{ isWAP } & & \multicolumn{2}{c}{ SWAP } \\
\cline { 2 - 3 } \cline { 5 - 6 } \cline { 5 - 6 } & $\mathrm{CR}$ & $\mathrm{CCR}$ & & $\mathrm{CR}$ & $\mathrm{CCR}$ \\
\hline Gate time $(\mathrm{ns})$ & 775.1 & 647.1 & & 1073.8 & 935.1 \\
Error $\left(10^{-2}\right)$ & $1.4(1)$ & $0.8(2)$ & & $3.5(9)$ & $2.0(6)$ \\
Coherence limit & 0.62 & 0.51 & & 0.85 & 0.74 \\
\hline \hline
\end{tabular}


(a)

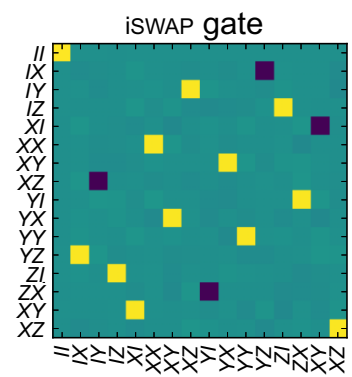

(b)

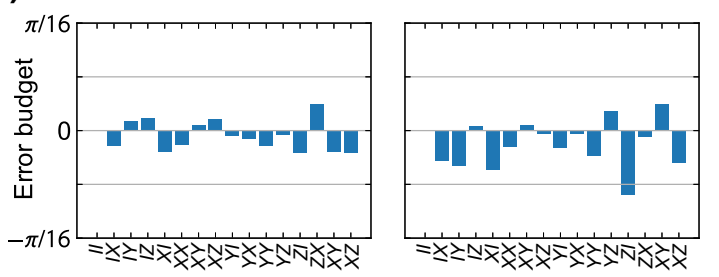

FIG. 4. (a) Pauli transfer matrices of isWAP (left) and SWAP (right) gates generated with CCR gates, evaluated by QPT experiments with measurement-error mitigation. (b) Generator intensities of rotation errors in isWAP (left) and SWAP (right) gates generated with CCR gates, extracted via a channel purification protocol.

errors are reconstructed; the ratio of the unitary error to the total gate error is $32 \%$ (isWAP) and 26\% (SWAP).

In general, QPT experiments are sensitive to state preparation and measurement (SPAM) errors, and we employ the measurement-error mitigation technique for accurate analysis. The average gate infidelities [48] are evaluated as $0.78 \times 10^{-2}$ (iSWAP) and $2.85 \times 10^{-2}$ (SWAP), which are almost comparable with the results of the IRB experiments. This indicates that our estimation of the unitary error is reliable, and that there is room for further reduction of the gate errors. Figure 4(b) shows the intensity of the generator for the reconstructed unitary error.

\section{SUMMARY AND DISCUSSION}

We propose and demonstrate a CCR gate, which is a two-qubit control scheme for dispersively coupled fixedfrequency transmon qubits. In the CCR gate, two qubits are simultaneously driven at the frequency of another qubit, and this operation induces both $Z X$ and $X Z$ terms in the effective Hamiltonian. For equivalent $Z X$ and $X Z$ interaction strengths, the CCR gate generates an entangling gate approximately locally equivalent to the isWAP rotation. Since the required microwave drive amplitudes are weaker than that for the CR gate, the CCR gate can suppress negative effects caused by the breaking of adiabatic conditions, such as drive-induced saturation and leakage from the computational basis. Our theoretical calculations show that iSWAP and SWAP gates implemented with the

CCR gate have half the leakage of those implemented with the CR gate for the same gate time.

In CCR gates, the drive amplitudes and drive frequencies are correlated by the drive-induced ac Stark shift caused by the bidirectional CR drives. We propose and demonstrate an efficient calibration technique to realize high-fidelity CCR gates to cope with the drive-induced Stark shift, along with a precise decomposition of isWAP and SWAP gates that nullifies the nonlocal error term appearing in the CCR-gate Hamiltonian. By using interleaved randomized benchmarking, we obtain a gate error of $0.8(2) \times 10^{-2}$ at a gate time of $647.1 \mathrm{~ns}$ for the isWAP gate, and $2.0(6) \times 10^{-2}$ at a gate time of $935.1 \mathrm{~ns}$ for the SWAP gate, which is better than the values for a conventional implementation based on $\mathrm{CR}$ gates.

Multimodal control pulses [19,45,46,49,50] can realize entangling gates that can potentially outperform existing entangling-gate implementations. This paper also shows an important achievement in terms of a reduction in the gate time and in leakage from the computational basis, which will pave the way for further device scaling with restricted qubit-to-qubit connectivity.

\section{ACKNOWLEDGMENTS}

We acknowledge fruitful discussions with Emily Pritchett, Ken X. Wei, Petar Jurcevic, Ikko Hamamura, Akhil Pratap Singh, Takanori Sugiyama, Yutaka Tabuchi, Shuhei Tamate, Atsushi Noguchi, and Yasunobu Nakamura. We acknowledge the researchers who created and supported the ibmq_bogota system, on which all data presented here were examined. K.H. was supported by a Japan Society for the Promotion of Science (JSPS) fellowship (JSPS KAKENHI Grant No. JP21J15221). We would like to thank Editage (www.editage.com) for English-language editing.

\section{APPENDIX A: EFFECTIVE-HAMILTONIAN MODEL OF CROSS-CROSS RESONANCE FOR A QUBIT MODEL}

In the qubit model, the anharmonicity is infinite, so the qubit subspace is perfectly isolated, and the Hamiltonian is given by

$$
\begin{aligned}
\mathcal{H}= & \omega_{0} \frac{Z I}{2}+\omega_{1} \frac{I Z}{2}+g X X \\
& +\Omega_{0} \cos \left(\omega_{d 0} t\right) X I+\Omega_{1} \cos \left(\omega_{d 1} t\right) I X,
\end{aligned}
$$

where, for simplicity, we assume a constant-amplitude drive $\Omega_{i}$ on the $X$ quadrature of the $i$ th qubit. Moving into the frame rotating at $\omega_{p}=\left(\omega_{d 0}+\omega_{d 1}\right) / 2$ via the unitary operator

$$
R=e^{-i \omega_{p} t(Z I / 2+I Z / 2)}
$$


gives

$$
\begin{aligned}
\mathcal{H}= & \left(\omega_{0}-\omega_{p}\right) \frac{Z I}{2}+\left(\omega_{1}-\omega_{p}\right) \frac{I Z}{2}+g\left(\frac{X X}{2}+\frac{Y Y}{2}\right) \\
& +\Omega_{0}\left(\cos \left(\omega_{m} t\right) \frac{X I}{2}-\sin \left(\omega_{m} t\right) \frac{Y I}{2}\right) \\
& +\Omega_{1}\left(\cos \left(\omega_{m} t\right) \frac{I X}{2}+\sin \left(\omega_{m} t\right) \frac{I Y}{2}\right),
\end{aligned}
$$

where $\omega_{m}=\left(\omega_{d 0}-\omega_{d 1}\right) / 2$. The Schrieffer-Wolff transformation [51] via the skew-Hermitian operator

$$
S=-i \frac{g}{\Delta}\left(\frac{X Y}{2}-\frac{Y X}{2}\right)
$$

gives the block-diagonal Hamiltonian as follows:

$$
\begin{aligned}
\mathcal{H} \sim & \left(\tilde{\omega}_{0}-\omega_{p}\right) \frac{Z I}{2}+\left(\tilde{\omega}_{1}-\omega_{p}\right) \frac{I Z}{2} \\
& +\Omega_{0}\left(\cos \left(\omega_{m} t\right) \frac{X I}{2}-\sin \left(\omega_{m} t\right) \frac{Y I}{2}\right) \\
& +\Omega_{1}\left(\cos \left(\omega_{m} t\right) \frac{I X}{2}+\sin \left(\omega_{m} t\right) \frac{I Y}{2}\right) \\
& +\frac{g \Omega_{0}}{\Delta}\left(\cos \left(\omega_{m} t\right) \frac{Z X}{2}-\sin \left(\omega_{m} t\right) \frac{Z Y}{2}\right) \\
& -\frac{g \Omega_{1}}{\Delta}\left(\cos \left(\omega_{m} t\right) \frac{X Z}{2}+\sin \left(\omega_{m} t\right) \frac{Y Z}{2}\right),
\end{aligned}
$$

where $\tilde{\omega_{0}}=\omega_{0}+g^{2} / \Delta$ and $\tilde{\omega_{1}}=\omega_{1}-g^{2} / \Delta$. Note that we truncate terms included in $O\left((g / \Delta)^{3}\right)$. Next, to consider the drive-induced Stark shift, moving into the rotating frame via the unitary operator

$$
R=e^{-i \omega_{m} t(Z I / 2-I Z / 2)}
$$

gives

$$
\begin{aligned}
\mathcal{H} \sim & \left(\tilde{\omega}_{0}-\omega_{d 0}\right) \frac{Z I}{2}+\left(\tilde{\omega}_{1}-\omega_{d 1}\right) \frac{I Z}{2} \\
& +\Omega_{0} \frac{X I}{2}+\Omega_{1} \frac{I X}{2} \\
& +\frac{g \Omega_{0}}{\Delta}\left(\cos \left(2 \omega_{m} t\right) \frac{Z X}{2}-\sin \left(2 \omega_{m} t\right) \frac{Z Y}{2}\right) \\
& -\frac{g \Omega_{1}}{\Delta}\left(\cos \left(2 \omega_{m} t\right) \frac{X Z}{2}+\sin \left(2 \omega_{m} t\right) \frac{Y Z}{2}\right) .
\end{aligned}
$$

The Schrieffer-Wolff transformation via the skewHermitian operator

$$
S=-i\left(\frac{\Omega_{0}}{\Delta_{0}} \frac{Y I}{2}+\frac{\Omega_{1}}{\Delta_{1}} \frac{I Y}{2}\right),
$$

where $\Delta_{i}=\tilde{\omega}_{i}-\omega_{d i}$, gives the block-diagonal Hamiltonian as follows:

$$
\begin{aligned}
\mathcal{H} \sim & \Delta_{0}\left(1+\frac{r_{0}^{2}}{2}\right) \frac{Z I}{2}+\Delta_{1}\left(1+\frac{r_{1}^{2}}{2}\right) \frac{I Z}{2} \\
& -\frac{\Omega_{0} r_{0}^{2}}{3} \frac{X I}{2}-\frac{\Omega_{1} r_{1}^{2}}{3} \frac{I X}{2} \\
& +\left\{\frac{g \Omega_{0}}{\Delta}\left(1-\frac{r_{0}^{2}+r_{1}^{2}}{2}\right)+\frac{g \Omega_{1}}{\Delta} r_{0} r_{1}\right\} \cos \left(2 \omega_{m} t\right) \frac{Z X}{2} \\
& -\left\{\frac{g \Omega_{1}}{\Delta}\left(1-\frac{r_{0}^{2}+r_{1}^{2}}{2}\right)+\frac{g \Omega_{0}}{\Delta} r_{0} r_{1}\right\} \cos \left(2 \omega_{m} t\right) \frac{X Z}{2} \\
& +\frac{g}{\Delta}\left(r_{1} \Omega_{1}-r_{0} \Omega_{0}\right) \cos \left(2 \omega_{m} t\right) \frac{X X}{2} \\
& +\frac{g}{\Delta}\left(r_{1} \Omega_{0}-r_{0} \Omega_{1}\right) \cos \left(2 \omega_{m} t\right) \frac{Z Z}{2} \\
& -\frac{g \Omega_{0}}{\Delta}\left(1-\frac{r_{0}^{2}}{2}\right) \sin \left(2 \omega_{m} t\right) \frac{Z Y}{2} \\
& -\frac{g \Omega_{1}}{\Delta}\left(1-\frac{r_{1}^{2}}{2}\right) \sin \left(2 \omega_{m} t\right) \frac{Y Z}{2} \\
& +\frac{g \Omega_{0}}{\Delta} r_{0} \sin \left(2 \omega_{m} t\right) \frac{X Y}{2} \\
& +\frac{g \Omega_{1}}{\Delta} r_{1} \sin \left(2 \omega_{m} t\right) \frac{Y X}{2},
\end{aligned}
$$

where $r_{i}=\Omega_{i} / \Delta_{i}$. Note that we truncate terms included in $O\left(\left(\Omega_{0} / \Delta\right)^{4}\right)$ and $O\left(\left(\Omega_{1} / \Delta\right)^{4}\right)$. We set the microwave drive frequencies $\omega_{d i}$ to

$$
\begin{aligned}
& \omega_{d 0}=\omega_{1}-\frac{\Omega_{0}^{2}}{2 \Delta}, \\
& \omega_{d 1}=\omega_{0}+\frac{\Omega_{1}^{2}}{2 \Delta}
\end{aligned}
$$

to satisfy the following conditions:

$$
\Delta_{0}\left(1+\frac{r_{0}^{2}}{2}\right)=-\Delta_{1}\left(1+\frac{r_{1}^{2}}{2}\right)=-2 \omega_{m}
$$

We can rewrite the Hamiltonian as follows:

$$
\begin{aligned}
\mathcal{H} \sim & \Delta\left\{1+\frac{1}{2}\left(\frac{\Omega_{0}}{\Delta}\right)^{2}\right\} \frac{Z I}{2}-\Delta\left\{1+\frac{1}{2}\left(\frac{\Omega_{1}}{\Delta}\right)^{2}\right\} \frac{I Z}{2} \\
& -\frac{\Omega_{0}}{3}\left(\frac{\Omega_{0}}{\Delta}\right)^{2} \frac{X I}{2}-\frac{\Omega_{1}}{3}\left(\frac{\Omega_{1}}{\Delta}\right)^{2} \frac{I X}{2} \\
& +\frac{g \Omega_{0}}{\Delta}\left\{1-\frac{1}{2}\left(\frac{\Omega_{0}}{\Delta}\right)^{2}-\frac{3}{2}\left(\frac{\Omega_{1}}{\Delta}\right)^{2}\right\} \cos \left(2 \omega_{m} t\right) \frac{Z X}{2}
\end{aligned}
$$




$$
\begin{aligned}
& -\frac{g \Omega_{1}}{\Delta}\left\{1-\frac{3}{2}\left(\frac{\Omega_{0}}{\Delta}\right)^{2}-\frac{1}{2}\left(\frac{\Omega_{1}}{\Delta}\right)^{2}\right\} \cos \left(2 \omega_{m} t\right) \frac{X Z}{2} \\
& -g\left\{\left(\frac{\Omega_{0}}{\Delta}\right)^{2}+\left(\frac{\Omega_{0}}{\Delta}\right)^{2}\right\} \cos \left(2 \omega_{m} t\right) \frac{X X}{2} \\
& -2 g \frac{\Omega_{0}}{\Delta} \frac{\Omega_{1}}{\Delta} \cos \left(2 \omega_{m} t\right) \frac{Z Z}{2} \\
& -\frac{g \Omega_{0}}{\Delta}\left\{1-\frac{1}{2}\left(\frac{\Omega_{0}}{\Delta}\right)^{2}\right\} \sin \left(2 \omega_{m} t\right) \frac{Z Y}{2} \\
& -\frac{g \Omega_{1}}{\Delta}\left\{1-\frac{1}{2}\left(\frac{\Omega_{1}}{\Delta}\right)^{2}\right\} \sin \left(2 \omega_{m} t\right) \frac{Y Z}{2} \\
& +g\left(\frac{\Omega_{0}}{\Delta}\right)^{2} \sin \left(2 \omega_{m} t\right) \frac{X Y}{2} \\
& +g\left(\frac{\Omega_{1}}{\Delta}\right)^{2} \sin \left(2 \omega_{m} t\right) \frac{Y X}{2} .
\end{aligned}
$$

Finally, moving into the rotating frame via the unitary operator

$$
R=e^{i 2 \omega_{m} t(Z I / 2-I Z / 2)}
$$

gives

$$
\begin{aligned}
\mathcal{H} \sim & -\frac{\Omega_{0}}{3}\left(\frac{\Omega_{0}}{\Delta}\right)^{2}\left\{\cos \left(2 \omega_{m} t\right) \frac{X I}{2}-\sin \left(2 \omega_{m} t\right) \frac{Y I}{2}\right\} \\
& -\frac{\Omega_{1}}{3}\left(\frac{\Omega_{1}}{\Delta}\right)^{2}\left\{\cos \left(2 \omega_{m} t\right) \frac{I X}{2}+\sin \left(2 \omega_{m} t\right) \frac{I Y}{2}\right\} \\
& +\frac{g \Omega_{0}}{\Delta}\left\{1-\frac{1}{2}\left(\frac{\Omega_{0}}{\Delta}\right)^{2}-\frac{3}{4}\left(\frac{\Omega_{1}}{\Delta}\right)^{2}\right\} \frac{Z X}{2} \\
& -\frac{3}{4} \frac{g \Omega_{0}}{\Delta}\left(\frac{\Omega_{1}}{\Delta}\right)^{2}\left\{\cos \left(4 \omega_{m} t\right) \frac{Z X}{2}+\sin \left(4 \omega_{m} t\right) \frac{Z Y}{2}\right\} \\
& -\frac{g \Omega_{1}}{\Delta}\left\{1-\frac{3}{4}\left(\frac{\Omega_{0}}{\Delta}\right)^{2}-\frac{1}{2}\left(\frac{\Omega_{1}}{\Delta}\right)^{2}\right\} \frac{X Z}{2} \\
& -\frac{3}{4} \frac{g \Omega_{1}}{\Delta}\left(\frac{\Omega_{0}}{\Delta}\right)^{2}\left\{\cos \left(4 \omega_{m} t\right) \frac{X Z}{2}-\sin \left(4 \omega_{m} t\right) \frac{Y Z}{2}\right\} \\
& -2 g \frac{\Omega_{0}}{\Delta} \frac{\Omega_{1}}{\Delta} \cos \left(2 \omega_{m} t\right) \frac{Z Z}{2} \\
& -g\left\{\left(\frac{\Omega_{0}}{\Delta}\right)^{2}+\left(\frac{\Omega_{0}}{\Delta}\right)^{2} \cos \left(4 \omega_{m} t\right)\right\} \cos \left(2 \omega_{m} t\right) \frac{X X}{2} \\
& +g\left(\frac{\Omega_{1}}{\Delta}\right)^{2} \cos \left(4 \omega_{m} t\right) \sin \left(2 \omega_{m} t\right) \frac{X Y}{2} \\
& g\left\{\left(\frac{\Omega_{0}}{\Delta}\right)^{2} \sin \left(2 \omega_{m} t\right)\right.
\end{aligned}
$$

$$
\begin{aligned}
& \left.+\left(\frac{\Omega_{1}}{\Delta}\right)^{2} \cos \left(2 \omega_{m} t\right) \sin \left(4 \omega_{m} t\right)\right\} \frac{Y X}{2} \\
& +g\left(\frac{\Omega_{1}}{\Delta}\right)^{2} \sin \left(4 \omega_{m} t\right) \sin \left(2 \omega_{m} t\right) \frac{Y Y}{2} .
\end{aligned}
$$

The above Hamiltonian $\mathcal{H}$ can be divided into a stationary term and a perturbation with a period $T=\pi / \omega_{m}$. We can calculate the effective Hamiltonian from the Magnus expansion as follows:

$$
\mathcal{H}_{\mathrm{eff}} \sim \frac{\operatorname{sgn}(\Delta) g \Omega_{0}}{\sqrt{\Delta^{2}+\Omega_{0}^{2}+\frac{3}{2} \Omega_{1}^{2}}} \frac{Z X}{2}-\frac{\operatorname{sgn}(\Delta) g \Omega_{1}}{\sqrt{\Delta^{2}+\frac{3}{2} \Omega_{0}^{2}+\Omega_{1}^{2}}} \frac{X Z}{2} .
$$

Note that the above notation for the effective Hamiltonian $\mathcal{H}_{\text {eff }}$ is a representation of a rotating frame moved from the laboratory frame using the unitary operator

$$
R=e^{-i\left(\Omega_{0}^{2} / 2 \Delta\right) t(Z I / 2)} e^{i\left(\Omega_{1}^{2} / 2 \Delta\right) t(I Z / 2)}
$$

\section{APPENDIX B: COMPARISON OF CCR AND CR GATES IN QUTRIT MODEL}

From Eq. (7), we can calculate the Cartan coefficients during a CCR gate in a two-qutrit system under the weakdrive condition $O(\Omega / \Delta)$ as follows:

$$
c(t)=\left(\frac{+g \Omega_{0}}{\Delta} \frac{\alpha_{0}}{\left(\alpha_{0}+\Delta\right)} t, \frac{-g \Omega_{1}}{\Delta} \frac{\alpha_{1}}{\left(\alpha_{1}-\Delta\right)} t, 0\right) .
$$

It can be seen that with the echo sequence shown in Figs. 3 (a) and 3(b), the execution time of isWAP and SWAP gates constructed with CCR gates is determined by the sum of the growth rates of the Cartan coefficients,

$$
v_{\text {ent }}=\frac{g}{\Delta}\left\{\left|\frac{\alpha_{0} \Omega_{0}}{\alpha_{0}+\Delta}\right|+\left|\frac{\alpha_{1} \Omega_{1}}{\alpha_{1}-\Delta}\right|\right\} .
$$

The application of the CCR drive to the qutrit system makes the adiabatic condition break and gives the maximum amount of leakage under the strong dispersive condition $O(g / \Delta)$ as follows:

$$
\xi_{\max }=\left(\frac{\Omega_{0}}{\alpha_{0}+\Delta}\right)^{2}+\left(\frac{\Omega_{1}}{\alpha_{1}-\Delta}\right)^{2}
$$

Note that we can also treat normal CR gates by setting $\Omega_{0}=0$ or $\Omega_{1}=0$. We assume that the best entangling gate minimizes the gate time while suppressing the maximum 
amount of leakage, which can be expressed as follows:

$$
\begin{array}{ll}
\operatorname{maximize} & v_{\mathrm{ent}} \\
\text { subject to } & \xi_{\max }=\xi
\end{array}
$$

Here, the latter constraint implies that the CCR drive amplitudes $\Omega_{0}$ and $\Omega_{1}$ satisfy an elliptic relation as follows:

$$
\left\{\begin{array}{l}
\Omega_{0}=\sqrt{\xi}\left(\alpha_{0}+\Delta\right) \cos \theta, \\
\Omega_{1}=\sqrt{\xi}\left(\alpha_{1}-\Delta\right) \sin \theta,
\end{array}\right.
$$

where $\theta \in[0,2 \pi]$ denotes an angle. Then, Eq. (B4) can be rewritten as follows:

$$
\text { maximize }\left|\alpha_{0} \cos \theta\right|+\left|\alpha_{1} \sin \theta\right| .
$$

One of the solutions is given as follows:

$$
\theta^{*}=\arctan \left(\frac{\alpha_{1}}{\alpha_{0}}\right)
$$

Finally, by substituting Eq. (B7) into Eq. (B5), we obtain the optimal ratio of CCR drive amplitudes as follows:

$$
\frac{\Omega_{1}}{\Omega_{0}}=\frac{\alpha_{1}\left(\alpha_{1}-\Delta\right)}{\alpha_{0}\left(\alpha_{0}+\Delta\right)}
$$

Then, we can compare the gate execution speeds of the CR and CCR gates for a fixed maximum amount of leakage $\xi$ as follows:

$$
\begin{aligned}
& \nu_{\mathrm{ent}}^{\mathrm{CR}(Q 0 \rightarrow Q 1)}=\frac{g}{\Delta} \sqrt{\frac{\xi}{2}} \alpha_{0}, \\
& \nu_{\mathrm{ent}}^{\mathrm{CR}(Q 1 \rightarrow Q 0)}=\frac{g}{\Delta} \sqrt{\frac{\xi}{2}} \alpha_{1},
\end{aligned}
$$

(a)

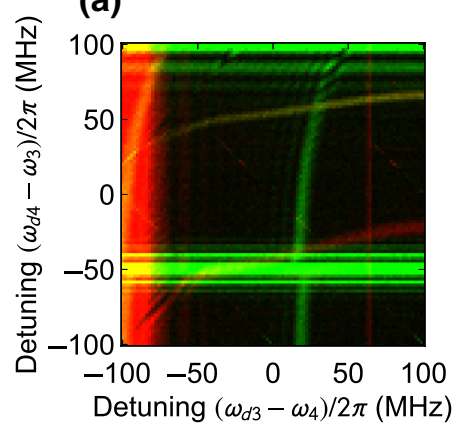

(b)

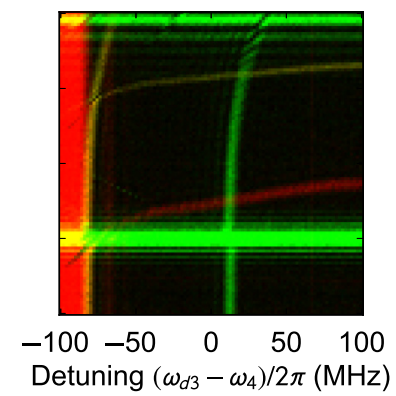

$$
\nu_{\mathrm{ent}}^{\mathrm{CCR}}=\frac{g}{\Delta} \sqrt{\frac{\xi}{2}} \sqrt{\alpha_{0}^{2}+\alpha_{1}^{2}}
$$

Similarly, the maximum amount of leakage for a fixed gate execution speed $v$ is written as follows:

$$
\begin{aligned}
& \xi_{\max }^{\mathrm{CR}(Q 0 \rightarrow Q 1)}=\frac{2}{\alpha_{0}^{2}}\left(\frac{\Delta v}{g}\right)^{2}, \\
& \xi_{\max }^{\mathrm{CR}(Q 1 \rightarrow Q 0)}=\frac{2}{\alpha_{1}^{2}}\left(\frac{\Delta v}{g}\right)^{2}, \\
& \xi_{\max }^{\mathrm{CCR}}=\frac{2}{\alpha_{0}^{2}+\alpha_{1}^{2}}\left(\frac{\Delta v}{g}\right)^{2} .
\end{aligned}
$$

From Eqs. (B11) and (B14), we can conclude that the CCR gate can achieve a speed $\sqrt{2}$ times faster or 2 times less leakage than the CR gate when the anharmonicity of the qubits is equal.

\section{APPENDIX C: CCR DRIVE FREQUENCIES WITH VARIOUS DRIVE AMPLITUDES}

As the CCR gate is driven, the eigenfrequencies of both qubits are shifted by the drive-induced Stark shift. Here, we observe the dependence of the operating point of the CCR drive frequency on the CCR drive strength. In this experiment, we first independently calibrate the $R_{Z X}(\pi / 4)$ and $R_{X Z}(\pi / 4)$ gates using CR gates with gate times of 145.8, 168.0, and 190.2 ns. Note that the pulse amplitude and gate time are inversely proportional. Next, while maintaining the respective drive amplitudes, CR drives from both directions are applied simultaneously at various drive frequencies. Figure 5 illustrates that the eigenfrequencies of $Q 3$ and $Q 4$ are functions of the gate time, which are

(c)
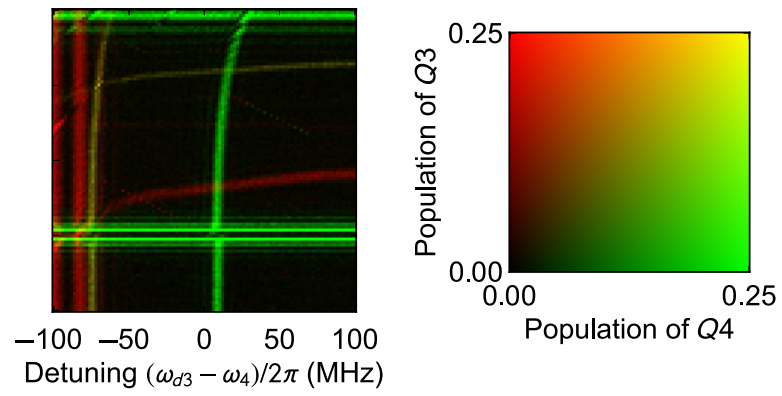

FIG. 5. Heatmaps of qubit excited-state populations measured after CCR gates with various frequency detunings from predetermined drive frequencies based on individual CR gate calibration. The measured populations of $Q 3$ and $Q 4$ are overlaid in the same plot. In each part, the vertical (horizontal) axis represents the detuning from the predetermined CR frequency of $Q 4(Q 3)$, corresponding to the frequency of the target qubit $Q 3(Q 4)$. The total duration of the $R_{Z X}(\pi / 4)$ gate is set to (a) 145.8, (b) 168.0, and (c) $190.2 \mathrm{~ns}$. 

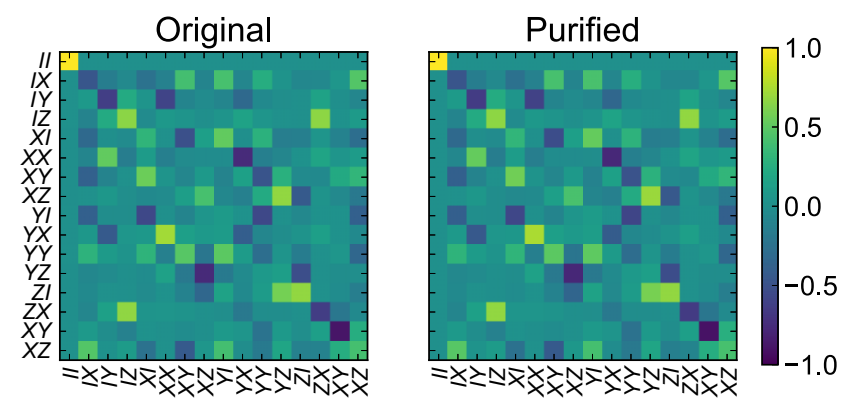

FIG. 6. Pauli transfer matrices of entangling gates generated by a CCR drive with a duration of $216.9 \mathrm{~ns}$ before (left) and after (right) channel purification, which roughly corresponds to the point shown by a star in Fig. 2(b).

redshifted and blueshifted, respectively, with respect to the drive amplitude.

In Fig. 5(a), it can be seen that the cross-resonance from $Q 4$ to $Q 3$ and the $g-f$ transition of $Q 4$ overlap. This suggests that the irradiation of $Q 3$ with the CR drive causes population leakage from the first excited state of $Q 3$ to the second excited state of $Q 4$. This phenomenon is not peculiar to CCR gates but also occurs in ordinary CR gates. We find that the two-dimensional drive-frequency sweep experiment provides important information to cope with these leakage errors.

\section{APPENDIX D: CHANNEL PURIFICATION}

To analyze the approximate Cartan coefficients of the experimentally generated quantum gate, we introduce a channel purification method that is derived from the McWeeny purification method [52]. In the channel purification method, the representation of a quantum channel $\mathcal{E}$ is first translated into a Choi representation according to the channel-state duality as follows:

$$
\rho_{\text {Choi }}=\sum_{i j} \chi_{i j}\left|P_{i}\right\rangle\left\langle P_{j}\right|
$$

where $\left|P_{i}\right\rangle$ is the vectorized $i$ th Pauli operator, and $\chi_{i j}$ is a coefficient of the corresponding Choi matrix of the channel. Here, the Chi matrix is defined as follows:

$$
\mathcal{E}(\rho)=\sum_{i j} \chi_{i j} P_{i} \rho P_{j}
$$

Next, we project the Choi matrix as follows:

$$
\rho_{\text {Choi }} \rightarrow\left|\psi_{\max }\right\rangle\left\langle\psi_{\max }\right|,
$$

where $\left|\psi_{\max }\right\rangle$ is the eigenvector of $\rho_{\text {choi }}$ with the maximum eigenvalue. The projected Choi matrix is translated back to the Kraus representation according to the channel-state duality. When the Choi matrix is projected to rank 1, there is only one Kraus operator $K$, and such a quantum channel satisfies the condition of complete positivity but is not trace-preserving. To derive a unitary operator that approximates the Kraus operator, we project the Kraus operator as follows:

$$
\begin{gathered}
K=M_{L} \operatorname{diag}\left\{r_{i} e^{i \theta_{i}}\right\} M_{R} \\
\rightarrow M_{L} \operatorname{diag}\left\{e^{i \theta_{i}}\right\} M_{L}^{-1},
\end{gathered}
$$

where $M_{L, R}$ is a regular matrix, and $r_{i} e^{i \theta_{i}}$ is a complex number. Figure 6 shows the Pauli transfer matrices before and after the channel purification. From Fig. 6, it is found that the Pauli transfer matrix does not change significantly as a result of the channel purification, which suggests that the target quantum channel is originally close to a unitary channel. The average gate infidelity between the Pauli transfer matrices before and after the channel purification is 0.015 , which includes residual SPAM errors that cannot be mitigated by measurement-error mitigation, and incoherent errors originating from decoherence while the CCR drive is applied.

Dynamical generator analysis [47], which is a similar method for reconstructing a unitary-matrix representation from a quantum channel, is not suitable for this study because it can be used only when the target quantum channel is included in branch 0 .
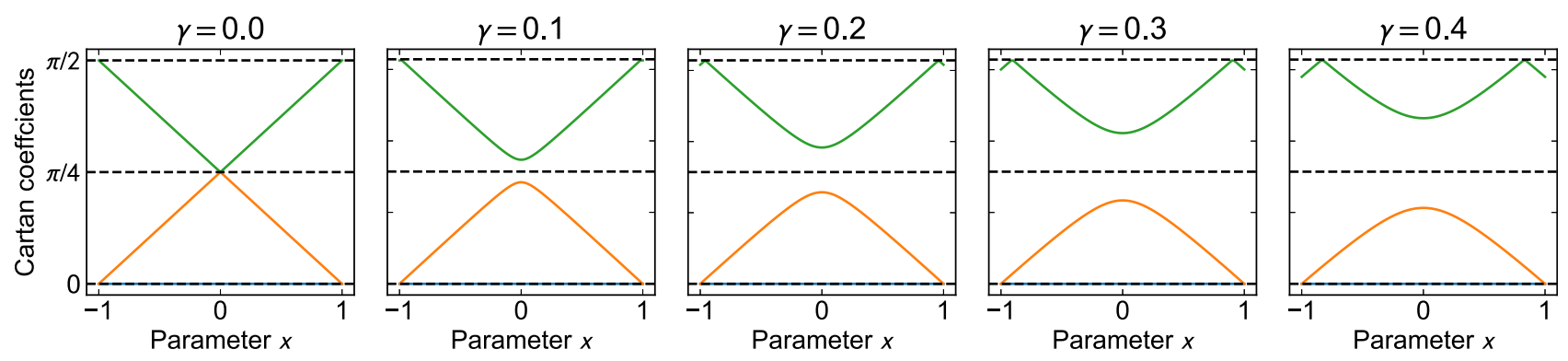

FIG. 7. Cartan coefficients of unitary gate generated by CCR drive with a static $Z Z$ error $\gamma Z Z$ ( $\gamma \in[0,0.4])$, while the ratio between the bidirectional CR drive amplitudes $(1-x):(1+x)$ is swept $(x \in[-1,1])$. Each line color corresponds to a Cartan coefficient; namely, there are three lines, for $c_{1}, c_{2}$, and $c_{3}$. 

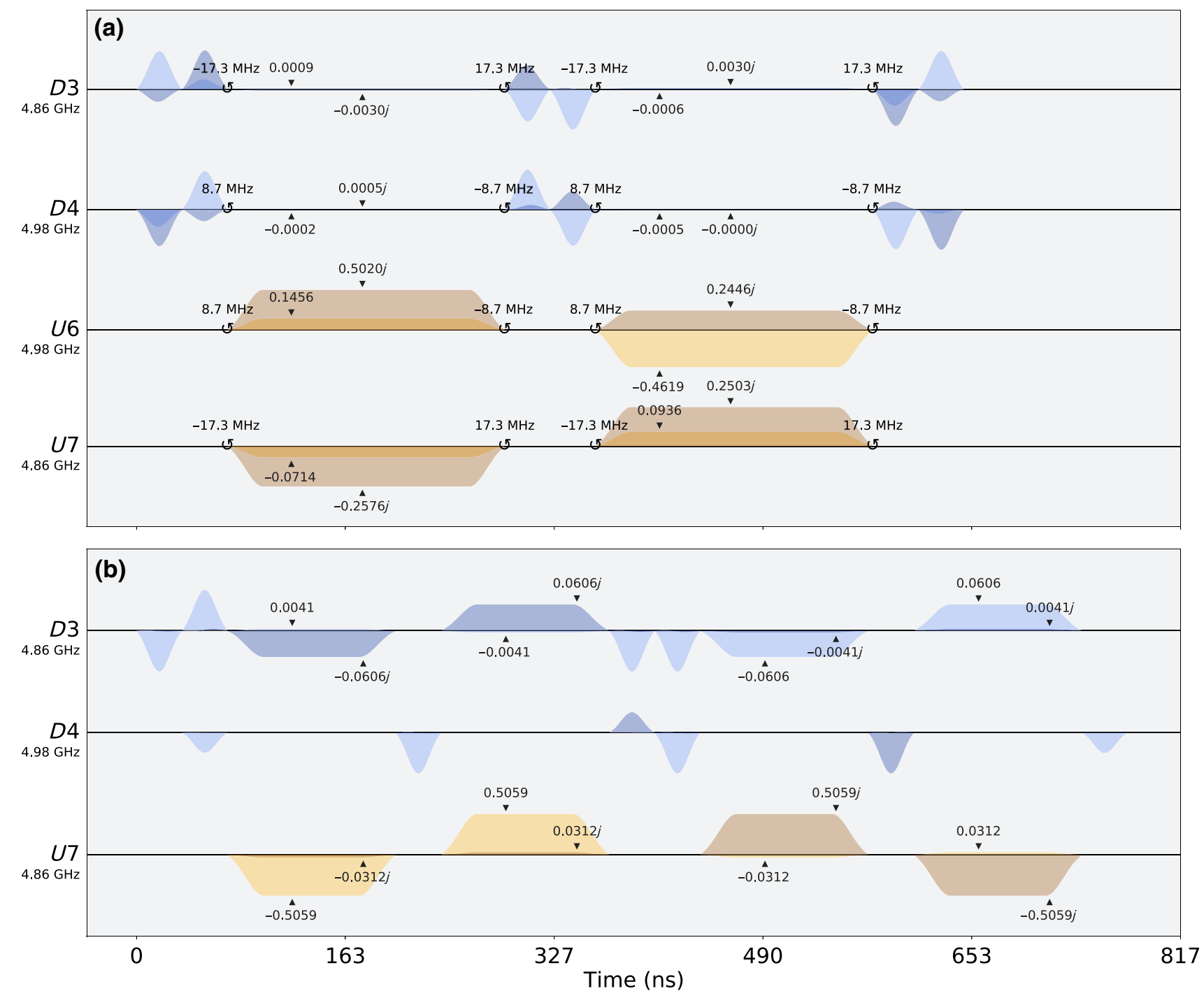

FIG. 8. Pulse schedules for isWAP gates with different implementations: (a) CCR gates and (b) TPCX gates. Each horizontal line corresponds to a pulse channel associated with a qubit in a specific frame. Channel names are shown on the left side, with the frequency assigned to the channel. Curly arrows indicate the frequency shift of the channel. These frequency shifts are introduced to correct driveinduced Stark shifts. The pulse amplitudes of the real and imaginary parts of the CCR and CR gates are also shown by triangle signs, where the ratio of the CR pulse amplitudes with which channels $U 6$ and $U 7$ are irradiated is designed to compensate for the different execution rates of the $\mathrm{CR}$ gates owing to finite anharmonicities.

\section{APPENDIX E: ANTICROSSING OF CARTAN COEFFICIENTS}

As mentioned in Sec. C, isWAP gates cannot be implemented with a single CCR gate, although Eq. (11) shows two nonzero Cartan coefficients. Here, we qualitatively estimate the imperfection of the CCR gate using a simplified Hamiltonian model. As discussed in Sec. B, the effective Hamiltonian of the CCR gate consists of $Z X$ and $X Z$ terms arising from the bidirectional cross-resonance drive. In addition, it is thought that $I X, I Y, X I$, and $Y I$ terms arise from classical crosstalk, and a $Z Z$ term arises when higher-order levels are considered. In principle, the terms derived from classical crosstalk can be eliminated by adding active cancellation drives. Therefore, we model the effective Hamiltonian as follows:

$$
H=\left(\frac{1-x}{2}\right) \frac{X Z}{2}+\left(\frac{1+x}{2}\right) \frac{Z X}{2}+\gamma \frac{Z Z}{2}
$$

where $x$ is a parameter corresponding to the ratio between the bidirectional CR drive amplitudes, and $\gamma$ is a perturbation coefficient for the static $Z Z$ term. We numerically calculate the Cartan coefficients for the unitary gate $U=e^{i \pi / 2 H}$ with various values of $x$ and $\gamma$. Figure 7 shows the results of the numerical simulation, where we calculate within $x \in[-1,1]$ and $\gamma \in[0,0.4]$. Recalling that an isWAP-type gate can be represented by Cartan 


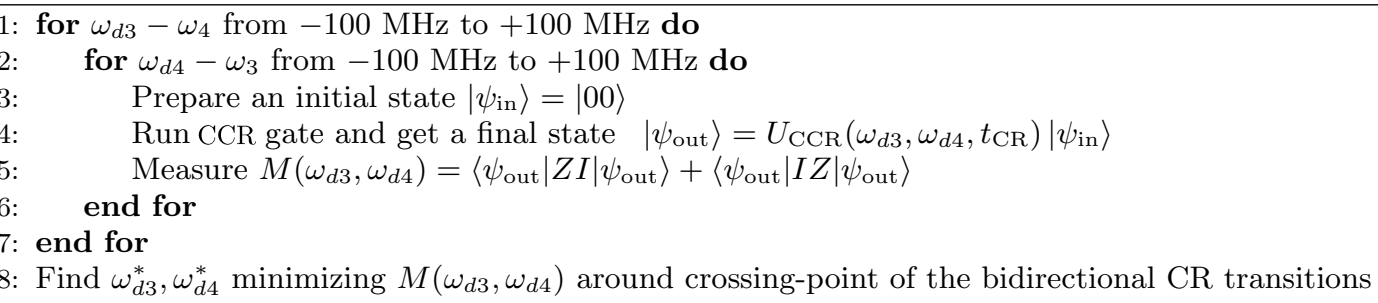

Algorithm 1. Calibration of CCR drive frequencies

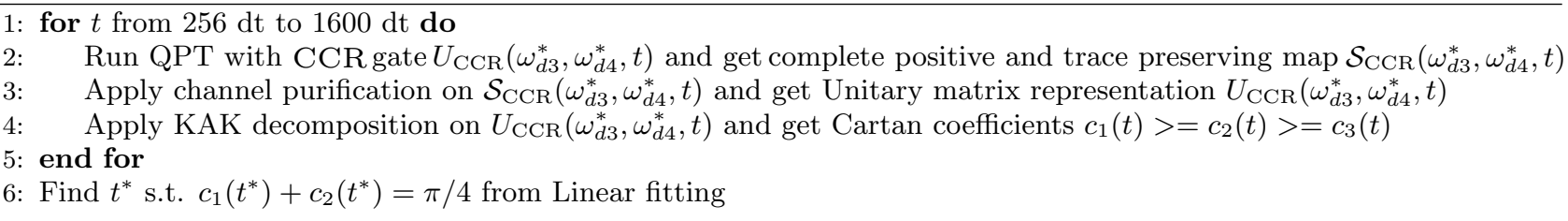

Algorithm 2. Calibration of CCR drive duration

coefficients $c_{1}=c_{2}$ and $c_{3}=0$, the nonzero $Z Z$ interaction, which is a dominant error source without a rotary echo [33], induces an anticrossing of two Cartan coefficients, yielding $c_{1} \neq c_{2}$. This simulation indicates that a high-fidelity isWAP gate cannot be implemented with a single CCR gate with qubits with a finite $Z Z$ error. On the other hand, suppression of the $Z Z$ interaction $[22,45,46]$ enables us to implement an isWAP gate with a single CCR gate and significantly shortens the gate time.

\section{APPENDIX F: PULSE SEQUENCES FOR ISWAP GATE}

Figure 8 shows a comparison of calibrated pulse schedules for isWAP gates with different implementations. The key component of the CCR gate is the bidirectional CR drive on control channel U6 (U7), which applies microwave tones to $Q 3(Q 4)$ in the frame of $Q 4(Q 3)$. Weak active-cancellation tones are simultaneously applied to the drive channels $D 3$ and $D 4$, which apply microwave tones in the frame of the associated qubits. Local operations are realized by two consecutive $\sqrt{X}$ pulses and three virtual$Z$ gates on $D 3$ and $D 4$ prepended and appended to the CCR gates. On the other hand, the standard isWAP decomposition generates a pulse sequence based on echoed $\mathrm{CR}$ gates, yielding a slightly longer schedule. In this decomposition, CR gates can be implemented with a rotary tone [53], which appears in $D 3$ and $D 4$ in parallel with CR tones with a comparable amplitude to those in $\sqrt{X}$ gates.

\section{APPENDIX G: PSEUDOCODES FOR CALIBRATION PROCEDURE OF THE CCR GATE}

To clarify the calibration procedure of a CCR gate, the relevant pseudocodes are summarized in Algorithms 1 and 2.
[1] Jared B. Hertzberg, Eric J. Zhang, Sami Rosenblatt, Easwar Magesan, John A. Smolin, Jeng-Bang Yau, Vivekananda P. Adiga, Martin Sandberg, Markus Brink, Jerry M. Chow, and Jason S. Orcutt, Laser-annealing Josephson junctions for yielding scaled-up superconducting quantum processors, arXiv:2009.00781 (2020).

[2] Maika Takita, Andrew W. Cross, A. D. Córcoles, Jerry M. Chow, and Jay M. Gambetta, Experimental Demonstration of Fault-Tolerant State Preparation with Superconducting Qubits, Phys. Rev. Lett. 119, 180501 (2017).

[3] Julian Kelly et al., State preservation by repetitive error detection in a superconducting quantum circuit, Nature 519, 66 (2015).

[4] Antonio D. Córcoles, Easwar Magesan, Srikanth J. Srinivasan, Andrew W. Cross, Matthias Steffen, Jay M. Gambetta, and Jerry M. Chow, Demonstration of a quantum error detection code using a square lattice of four superconducting qubits, Nat. Commun. 6, 6979 (2015).

[5] Rami Barends, Julian Kelly, Anthony Megrant, Andrzej Veitia, Daniel Sank, Evan Jeffrey, Ted C. White, Josh Mutus, Austin G. Fowler, and Brooks Campbell et al., Superconducting quantum circuits at the surface code threshold for fault tolerance, Nature 508, 500 (2014).

[6] Maika Takita, Antonio D. Córcoles, Easwar Magesan, Baleegh Abdo, Markus Brink, Andrew Cross, Jerry M. Chow, and Jay M. Gambetta, Demonstration of WeightFour Parity Measurements in the Surface Code Architecture, Phys. Rev. Lett. 117, 210505 (2016).

[7] Frank Arute, Kunal Arya, Ryan Babbush, Dave Bacon, Joseph C. Bardin, Rami Barends, Rupak Biswas, Sergio Boixo, Fernando GSL Brandao, and David A. Buell et al., Quantum supremacy using a programmable superconducting processor, Nature 574, 505 (2019).

[8] Petar Jurcevic, Ali Javadi-Abhari, Lev S. Bishop, Isaac Lauer, Daniela Borgorin, Markus Brink, Lauren Capelluto, Oktay Gunluk, Toshinari Itoko, and Naoki Kanazawa et al., 
Demonstration of quantum volume 64 on a superconducting quantum computing system, Quantum Sci. Technol. 6, 025020 (2021).

[9] Andrew W. Cross, Lev S. Bishop, Sarah Sheldon, Paul D. Nation, and Jay M. Gambetta, Validating quantum computers using randomized model circuits, Phys. Rev. A 100, 032328 (2019).

[10] Alexandre Blais, Alexander Maassen van den Brink, and Alexandre M. Zagoskin, Tunable Coupling of Superconducting Qubits, Phys. Rev. Lett. 90, 127901 (2003).

[11] Travis Hime, P. A. Reichardt, B. L. T Plourde, T. L. Robertson, C-E. Wu, AV Ustinov, and John Clarke, Solid-state qubits with current-controlled coupling, Science 314, 1427 (2006).

[12] A. O. Niskanen, K Harrabi, F Yoshihara, Y Nakamura, S Lloyd, and J. S. Tsai, Quantum coherent tunable coupling of superconducting qubits, Science 316, 723 (2007).

[13] J. Stehlik, D. M. Zajac, D. L. Underwood, T. Phung, J. Blair, S. Carnevale, D. Klaus, G. A. Keefe, A. Carniol, M. Kumph, Matthias Steffen, and O. E. Dial, Tunable coupling architecture for fixed-frequency transmons, arXiv:2101.07746 (2021).

[14] Chad Rigetti and Michel Devoret, Fully microwave-tunable universal gates in superconducting qubits with linear couplings and fixed transition frequencies, Phys. Rev. B 81, 134507 (2010).

[15] P. C. De Groot, J. Lisenfeld, R. N. Schouten, S. Ashhab, A. Lupaşcu, C. J. P. M. Harmans, and J. E. Mooij, Selective darkening of degenerate transitions demonstrated with two superconducting quantum bits, Nat. Phys. 6, 763 (2010).

[16] S. Poletto, Jay M. Gambetta, Seth T. Merkel, John A. Smolin, Jerry M. Chow, A. D. Córcoles, George A. Keefe, Mary B. Rothwell, J. R. Rozen, D. W. Abraham, Chad Rigetti, and M. Steffen, Entanglement of two Superconducting Qubits in a Waveguide Cavity via Monochromatic Two-Photon Excitation, Phys. Rev. Lett. 109, 240505 (2012).

[17] Jerry M. Chow, Jay M. Gambetta, Andrew W. Cross, Seth T. Merkel, Chad Rigetti, and M. Steffen, Microwave-activated conditional-phase gate for superconducting qubits, New J. Phys. 15, 115012 (2013).

[18] S. Krinner, P. Kurpiers, B. Royer, P. Magnard, I. Tsitsilin, J.-C. Besse, A. Remm, A. Blais, and A. Wallraff, Demonstration of an All-Microwave Controlled-Phase Gate between Far-Detuned Qubits, Phys. Rev. Appl. 14, 044039 (2020).

[19] Atsushi Noguchi, Alto Osada, Shumpei Masuda, Shingo Kono, Kentaro Heya, Samuel Piotr Wolski, Hiroki Takahashi, Takanori Sugiyama, Dany Lachance-Quirion, and Yasunobu Nakamura, Fast parametric two-qubit gates with suppressed residual interaction using the second-order nonlinearity of a cubic transmon, Phys. Rev. A 102, 062408 (2020).

[20] Sarah Sheldon, Easwar Magesan, Jerry M. Chow, and Jay M. Gambetta, Procedure for systematically tuning up cross-talk in the cross-resonance gate, Phys. Rev. A 93, 060302(R) (2016).

[21] Jaseung Ku, Xuexin Xu, Markus Brink, David C. McKay, Jared B. Hertzberg, Mohammad H. Ansari, and B. L. T. Plourde, Suppression of Unwanted ZZ Interactions in a
Hybrid Two-Qubit System, Phys. Rev. Lett. 125, 200504 (2020).

[22] A. Kandala, K. X. Wei, S. Srinivasan, E. Magesan, S. Carnevale, G. A. Keefe, D. Klaus, O. Dial, and D. C. McKay, Demonstration of a high-fidelity cnot for fixedfrequency transmons with engineered $\mathrm{ZZ}$ suppression, arXiv:2011.07050 (2020).

[23] Jerry M. Chow, A. D. Córcoles, Jay M. Gambetta, Chad Rigetti, B. R. Johnson, John A. Smolin, J. R. Rozen, George A. Keefe, Mary B. Rothwell, Mark B. Ketchen, and M. Steffen, Simple All-Microwave Entangling Gate for FixedFrequency Superconducting Qubits, Phys. Rev. Lett. 107, 080502 (2011).

[24] Moein Malekakhlagh, Easwar Magesan, and David C. McKay, First-principles analysis of cross-resonance gate operation, Phys. Rev. A 102, 042605 (2020).

[25] Vinay Tripathi, Mostafa Khezri, and Alexander N. Korotkov, Operation and intrinsic error budget of a twoqubit cross-resonance gate, Phys. Rev. A 100, 012301 (2019).

[26] Thomas Alexander, Naoki Kanazawa, Daniel J. Egger, Lauren Capelluto, Christopher J. Wood, Ali Javadi-Abhari, and David C. McKay, Qiskit pulse: Programming quantum computers through the cloud with pulses, Quantum Sci. Technol. 5, 044006 (2020).

[27] Shelly Garion, Naoki Kanazawa, Haggai Landa, David C. McKay, Sarah Sheldon, Andrew W. Cross, and Christopher J. Wood, Experimental implementation of non-Clifford interleaved randomized benchmarking with a controlled-s gate, arXiv:2007.08532 (2020).

[28] J. Emerson, R. Alicki, and K. Życzkowski, Scalable noise estimation with random unitary operators, J. Opt. B: Quantum Semiclass. opt. 7, S347 (2005).

[29] E. Knill, D. Leibfried, R. Reichle, J. Britton, R. B. Blakestad, J. D. Jost, C. Langer, R. Ozeri, S. Seidelin, and D. J. Wineland, Randomized benchmarking of quantum gates, Phys. Rev. A 77, 012307 (2008).

[30] E. Magesan, J. M. Gambetta, and J. Emerson, Scalable and Robust Randomized Benchmarking of Quantum Processes, Phys. Rev. Lett. 106, 180504 (2011).

[31] Easwar Magesan and Jay M. Gambetta, Effective hamiltonian models of the cross-resonance gate, Phys. Rev. A 101, 052308 (2020).

[32] Paul Watts, Maurice O';Connor, and Jiří Vala, Metric structure of the space of two-qubit gates, perfect entanglers and quantum control, Entropy 15, 1963 (2013).

[33] Jay Gambetta, Alexandre Blais, David I. Schuster, Andreas Wallraff, L. Frunzio, J. Majer, Michel H. Devoret, Steven M. Girvin, and Robert J. Schoelkopf, Qubit-photon interactions in a cavity: Measurement-induced dephasing and number splitting, Phys. Rev. A 74, 042318 (2006).

[34] Andre Schneider, Jochen Braumüller, Lingzhen Guo, Patrizia Stehle, Hannes Rotzinger, Michael Marthaler, Alexey V. Ustinov, and Martin Weides, Local sensing with the multilevel ac stark effect, Phys. Rev. A 97, 062334 (2018).

[35] Kentaro Heya and Naoki Kanazawa, Pauli gate error amplification for sophisticated quantum gate calibration, Bull. Am. Phys. Soc. 66, M33.1 (2021). 
[36] Neereja Sundaresan, Isaac Lauer, Emily Pritchett, Easwar Magesan, Petar Jurcevic, and Jay M. Gambetta, Reducing unitary and spectator errors in cross resonance with optimized rotary echoes, PRX Quantum 1, 020318 (2020).

[37] P. J. Leek, S. Filipp, P. Maurer, M. Baur, R. Bianchetti, J. M. Fink, M. Göppl, L. Steffen, and A. Wallraff, Using sideband transitions for two-qubit operations in superconducting circuits, Phys. Rev. B 79, 180511(R) (2009).

[38] Filip B. Maciejewski, Zoltán Zimborás, and Michał Oszmaniec, Mitigation of readout noise in near-term quantum devices by classical post-processing based on detector tomography, Quantum 4, 257 (2020).

[39] Yanzhu Chen, Maziar Farahzad, Shinjae Yoo, and TzuChieh Wei, Detector tomography on IBM quantum computers and mitigation of an imperfect measurement, Phys. Rev. A 100, 052315 (2019).

[40] Hyeokjea Kwon and Joonwoo Bae, A hybrid quantumclassical approach to mitigating measurement errors in quantum algorithms, IEEE Trans. Comput. 70, 1401 (2021).

[41] Suguru Endo, Zhenyu Cai, Simon C. Benjamin, and Xiao Yuan, Hybrid quantum-classical algorithms and quantum error mitigation, J. Phys. Soc. Jpn. 90, 032001 (2021).

[42] M. Mohseni, A. T. Rezakhani, and D. A. Lidar, Quantumprocess tomography: Resource analysis of different strategies, Phys. Rev. A 77, 032322 (2008).

[43] Deanna M. Abrams, Nicolas Didier, Blake R. Johnson, Marcus P. da Silva, and Colm A. Ryan, Implementation of XY entangling gates with a single calibrated pulse, Nat. Electron. 3, 744 (2020).

[44] Malcolm Carroll, Sami Rosenblatt, Petar Jurcevic, Isaac Lauer, and Abhinav Kandala, Dynamics of superconducting qubit relaxation times, arXiv:2105.15201 (2021).

[45] Bradley K. Mitchell, Ravi K. Naik, Alexis Morvan, Akel Hashim, John Mark Kreikebaum, Brian Marinelli, Wim Lavrijsen, Kasra Nowrouzi, David I. Santiago, and Irfan Siddiqi, Hardware-efficient microwave-activated tunable coupling between superconducting qubits, arXiv:2105.05384 (2021).

[46] K. X. Wei, E. Magesan, I. Lauer, S. Srinivasan, D. F. Bogorin, S. Carnevale, G. A. Keefe, Y. Kim, D. Klaus, W. Landers, N. Sundaresan, C. Wang, E. J. Zhang, M. Steffen, O. E. Dial, D. C. McKay, and A. Kandala, Quantum crosstalk cancellation for fast entangling gates and improved multi-qubit performance, arXiv:2106.00675 (2021).

[47] Takanori Sugiyama, Shinpei Imori, and Fuyuhiko Tanaka, Self-consistent quantum tomography with regularization, Phys. Rev. A 103, 062615 (2021).

[48] Michael A. Nielsen, A simple formula for the average gate fidelity of a quantum dynamical operation, Phys. Lett. A 303, 249 (2002).

[49] Yosep Kim, Alexis Morvan, Long B. Nguyen, Ravi K. Naik, Christian Jünger, Larry Chen, John Mark Kreikebaum, David I. Santiago, and Irfan Siddiqi, High-fidelity itoffoli gate for fixed-frequency superconducting qubits, arXiv:2108.10288 (2021).

[50] Xiu Gu, Jorge Fernández-Pendás, Pontus Vikstål, Tahereh Abad, Christopher Warren, Andreas Bengtsson, Giovanna Tancredi, Vitaly Shumeiko, Jonas Bylander, Göran Johansson, and Anton Frisk Kockum, Fast multi-qubit gates through simultaneous two-qubit gates, arXiv:2108.11358 (2021).

[51] J. R. Schrieffer and P. A. Wolff, Relation between the Anderson and Kondo hamiltonians, Phys. Rev. 149, 491 (1966).

[52] R. McWeeny and Charles Alfred Coulson, The density matrix in self-consistent field theory I. iterative construction of the density matrix, ' Proc. Math. Phys. Eng. Sci. 235, 496 (1956).

[53] Neereja Sundaresan, Isaac Lauer, Emily Pritchett, Easwar Magesan, Petar Jurcevic, and Jay M. Gambetta, Reducing unitary and spectator errors in cross resonance with optimized rotary echoes, PRX Quantum 1, 020318 (2020). 